UDK 524.57

PASC 98.38.Cp, 98.38.Am, 95.30.Qd

\title{
ON SATURATION OF BETATRON ACCELERATION OF DUST PARTICLES BEHIND SHOCK FRONTS
}

\author{
L. V. Kostyukova and V. V. Prudskikh \\ Faculty of Physics of Southern Federal University, Rostov on Don, Russia \\ Yu. A. Shchekinov \\ Faculty of Physics of Southern Federal University, Rostov on Don, Russia and \\ Special Astrophysical Observatory of RAS, \\ Nizhny Arkhyz, Karachai-Cherkessiya, Russia
}

(Received 22.06.2009 J.; Revised 10.08.2009 J.)

\begin{abstract}
Possible saturation of betatron acceleration of dust particles behind strong shock fronts from supernovae is considered. It is argued that the efficiency of the nonthermal dust destruction should be substantially lower than the value estimated from a traditional description of betatron acceleration of dust grains behind radiative shock waves. The inhibition of the nonthermal destruction can be connected with the mirror instability developed in the dust component behind strong shocks with the velocity 3 times exceeding the Alfvén speed. The instability develops on characteristic time scales much shorter the age of a supernova remnant, thus its influence on the efficiency of dust destruction can be substantial: in the range of shock velocities $100 \mathrm{~km} \mathrm{~s}^{-1}<v_{s}<300 \mathrm{~km} \mathrm{~s}^{-1}$ the destruction efficiency can be an order of magnitude lower that normally estimated.
\end{abstract}

\section{INTRODUCTION}

At present it is commonly accepted that dust grains are efficiently destroyed behind shock fronts from supernovae [1,2]. A rough estimate of the dust destruction rate in the Galaxy can be obtained from the following simple consideration [2]: the total mass of the dust destroyed in unite time is $\dot{M}_{d}=\nu_{S N} \eta M / \zeta$, where $\nu_{S N}$ is the Galactic supernova rate, $\eta$ is the efficiency of dust destruction by a shock with a given velocity, $M$ is the mass of supernova shell with a given velocity; the kinetic energy of the shell is assumed by order of magnitude to be equal to the explosion energy $E \sim M v^{2} / 2$. It is commonly assumed that dust grains most efficiently are destroyed by the shock waves with the velocities $v \geq 100 \mathrm{~km} \mathrm{~s}^{-1}$ - at lower velocities the destruction efficacy drops exponentially [1]. The fraction of destroyed dust behind the shock with the velocity $v_{s, 0.4}=100 \mathrm{~km} \mathrm{~s}^{-1}$ is around $\eta \simeq 0.4$, hence the total rate of the dust mass decrease in the Galaxy is estimated as

$$
\dot{M}_{d} \sim 0.8 \nu_{S N} \frac{E_{S N}}{\zeta v_{s, 0.4}^{2}}
$$

where $E_{S N}=10^{51} \mathrm{erg}$ is the explosion energy, $\zeta=100-200$ is the mean gas-to-dust mass ratio. The characteristic time of dust destruction is

$$
\tau_{d, d}=\frac{M_{d}}{\dot{M}_{d}}=1.25 \frac{M_{g} v_{s, 0.4}^{2}}{E_{S N} \nu_{S N}} \sim 10^{8} \text { year }
$$

where $M_{g}=5 \times 10^{9} M_{\odot}$, the gas mass in the Galaxy, the suparnova rate is assumed to be $\nu_{S N}=1 / 100$ year $^{-1}$.

It is obvious that this value of $\tau_{d, d}$ is unacceptably short in comparison with the time of dust formation (replenishment) in the Galaxy. Indeed, the total rate of dust injection into the interstellar medium (ISM) from stellar sources (including supernovae) is $\leq 3 \times$ 
$10^{-11} M_{\odot} \mathrm{pc}^{-2}$ year $^{-1}[3-5]$. Assuming now the total dust mass in the Galaxy $\sim 5 \times 10^{7} M_{\odot}$, one can estimate the replenishment rate is $\tau_{d, f} \sim 10^{9}$ years. In such conditions the total dust mass in the Galaxy must be an order of magnitude lower than the observed value, i.e. $\sim 5 \times 10^{5} M_{\odot}$.

Moreover, it is well known that the abundances of refractory elements in gas phase of the ISM are always substantially lower than solar values. In other words such elements are "depleted" in gas phase. This depletion is connected with their condensation on the surface of dust grains. Even in the diffuse rarefied phase $\left(n \simeq 0.05 \mathrm{~cm}^{-3}\right)$ the depletion factor can be as high as $90 \%$ for $\mathrm{Fe}, \mathrm{Ca}$, Ti, i.e. the fraction of these elements in gas phase is $10 \%$ or lower, while in more dense regions $\left(n \simeq 3 \mathrm{~cm}^{-3}\right)$ the fraction of Fe in gas phase is around $1 \%$, and the fraction of $\mathrm{Ca}$ and $\mathrm{Ti}$ is onlu $0.1 \%$ [6,7]. This means that destruction of dust (and as a consequence removal of metals from condensed phase into gaseous) should be considerably slower process than their formation, i.e. condensation of metals on to the solid (dust) phase. In particular, for the diffuse ISM the following interrelation between the characteristic dust destruction $\tau_{d, d}$ and formation $\tau_{d, f}$ times: $\tau_{d, f}=0.1 \tau_{d, d}$. In general, the condition $\tau_{d, f} \ll \tau_{d, d}$ has to be always fulfilled, though at present the mechanisms able to maintain this condition are unknown.

One of the possibilities is connected with that a considerable dust mass is confined in dense atomic and molecular clouds. When a shock wave falls on to a dense cloud its velocity inside the cloud decreases as $v_{s, c} \sim v_{s, i} \sqrt{\rho_{i} / \rho_{c}}$, where indices $c$ and $i$ belong to the cloud and the intercloud medium, respectively. Even for HI clouds with density only one or one and a half orders higher than in the intercloud medium the shock velocity decreases by factor $3-5$, so that dust remains undestroyed behind such shock waves. Therefore, only dust in the diffuse intercloud gas is destroyed by supernovae shocks. With this circumstance accounted the above estimate can be rewritten as follows

$$
\dot{M}_{d} \sim 0.8 \mu_{i} \nu_{S N} \frac{E_{S N}}{\zeta v_{s, 0.4}^{2}}
$$

Here $\mu_{i}=\rho_{i}\left(1-f_{c}\right) / \rho_{c} f_{c}$ is the mass fraction contained in the intercloud medium, $f_{c}$ is the volume filling factor of clouds:

$$
f_{c}=\frac{4}{3} \pi V_{\mathrm{MC}}^{-1} \int R_{c}^{3} \frac{d N}{d M} d M
$$

where the cloud mass spectrum $d N / d M \propto M^{-1.7}$ in the mass range $10^{-4} M_{\odot}<M<$ $10^{5} M_{\odot}$ is assumed $[8,9]$; the estimate gives $\mu_{i} \sim 0.1$. As a result, the dust destruction time increases as $\tau_{d, d} \sim 2 \times 10^{8} / \mu_{i}$ and becomes comparable to the Galactic dust production time. At the same time the necessary interrelation $\tau_{d, f} \ll \tau_{d, d}$ remains unexplained.

Apparently, one has to assume that in such conditions condensation of metals on to solid (dust) phase should occur directly in the ISM. A low abundance of deuterium in gas phase of the local interstellar medium seems to favor this conclusion. It is argued in [10] that the observed relative abundances of deuterium and oxygen indicate that deuterium is frozen-out on dust grains: the characteristic time for deuterium to freeze out is estimated in [10] to be around several Myr in the conditions of diffuse interstellar clouds.

The latter means apparently that dust grains escape destruction by supernovae shocks. Indeed, all known data about the depletion of deuterium belongs to the nearest vicinity of Sun restricted by distances of 100-150 pc. The characteristic interval between successful explosions of supernovae in this volume is around $5 \times 10^{5}$ year [11] - an order of magnitude shorter than the time of deuterium freezing on the dust surface. Therefore deuterium can be depleted in gas phase of the local ISM provided only that SNe shocks do not destroy dust grains. 
In this paper we present arguments favoring that plasma instabilities behind shock waves prevent an efficient acceleration of charged dust particles by betatron mechanism, and thus inhibit their destruction. An essential circumstance here is that the dust component can change its dynamics only on times corresponding to the inverse dust cyclotron frequency $\omega_{d}$. This requires the presence of plasma oscillations with frequences close to $\omega_{d}$ in the whole spectrum, which seems plausible only under description of the dust as a separate dynamical component. Therefore, in this paper we describe instabilities in the interstellar plasma behind strong shock waves caused by the presence of the charged dust component.

In Sect. 2 we formulate the equation of motions of a three-fluid (ions, electrons and charged dust) interstellar plasma, in Sect. 3 we derive the dispersion equation for small oscillations in such a plasma, in Sect. 4 the dispersion equation is analyzed and the conditions for instabilities are discussed, Sect. 5 summarizes the results.

\section{EQUATIONS OF MOTION}

An efficient destruction of dust particles behind shock fronts is determined by their betatron acceleration in the course of plasma compression on radiative stages $[2$, 12]. Namely, during a radiative cooling and an accompanying compression of plasma magnetic field behind the shock front grows as $B \propto \rho$, what in turn leads to a proportional increase of the square of the transversal momentum of dust particles $p_{\perp}^{2} \propto B$. Consequently the efficiency of dust destruction increases by more than an order of magnitude for the shocks with velocities $100 \mathrm{~km} \mathrm{~s}^{-1}<$ $<v_{s}<300 \mathrm{~km} \mathrm{~s}^{-1}$. In these estimates the structure of the post-shock flow, in particular, the magnetic field being parallel to the front, is assumed implicitly to be stable and invariable over the whole post-shock region. At the same time, one can think that growing plasma instabilities are able to violate the symmetry of the flow and moreover to make the flow irregular. As a result, plasma regions with a decreased magnetic field and the correspondingly decreased dust velocities can arise, which result in an inhibition of the dust destruction efficiency.

One of the instabilities which can cause violation of a regular magneto-hydrodynamic flow is the mirror instability of an anisotropic plasma with the transversal temperature exceeding the parallel one: $T_{\perp} \gg T_{\|}$. In the conditions behind shock fronts anisotropy of the dust component emerges naturally: after crossing a shock front with a parallel magnetic field a dust grain gains the transerval velocity in the restframe connected with the front close to the shock velocity, while the longitudinal velocity remains equal to a much lower velocity of random dust motions in the ISM. Below we consider a possibility for the mirror instability to develop in such conditions.

Let us describe the interstellar plasma by the equations [13]

$$
\partial_{t} n_{\alpha}+\nabla \cdot\left(n_{\alpha} \mathbf{u}_{\alpha}\right)=0
$$

where $\alpha=i, e, d$ corresponds to the ions, electrons and dust particles. Conservation of the momentum is described by the equations

$$
\begin{gathered}
\partial_{t}\left(n_{\alpha} \mathbf{u}_{\alpha}\right)+\nabla \cdot\left(n_{\alpha} \mathbf{u}_{\alpha} \mathbf{u}_{\alpha}\right)= \\
=-\frac{1}{m_{\alpha}} \nabla p_{\alpha}-\frac{n_{\alpha} \tilde{Z}_{\alpha} e}{m_{\alpha}}\left(\nabla \Phi-\frac{1}{c}\left[\mathbf{v}_{\alpha} \mathbf{B}\right]\right),
\end{gathered}
$$

which should be complemented by the equations

$$
\Delta \Phi=-4 \pi e \sum \tilde{Z}_{\alpha} n_{\alpha}
$$


and

$$
\nabla \times \mathbf{B}=\frac{4 \pi e}{c} \sum \tilde{Z}_{\alpha} n_{\alpha} \mathbf{v}_{\alpha}
$$

as well as the equation for curlE; here $\tilde{Z}_{e}=-1, \tilde{Z}_{i}=+1$ and $\tilde{Z}_{d}=-Z_{d}$, so that in typical conditions with negative dust grains the quasi-neutrality equation has the form $n_{i}=n_{e}+\tilde{Z}_{d} n_{d}$.

Let us consider plasma behind the shock in the comoving (moving with the plasma) restframe. Let us consider also the configuration with magnetic field poins parallel to $z$ axis (along the shock front), while the wavevector has the components $\mathbf{k}=\left(k_{\perp}, 0, k_{\|}\right)$. In this work we neglect the effects connected with perturbations of the front. Therefore our results formally are valid far from the front - stability of the shock front in such a multi-component plasma deserves a separate consideration.

Let us linearize the system (5)-(8) in projections for each plasma components. We will restrict consideration by low-frequency electromagnetic waves with the phase velocity satisfying the inequality

$$
v_{T i} \ll \omega / k \ll v_{T e},
$$

where $v_{T e}$ and $v_{T i}$ are thermal velocities of the electrons and ions, respectively. For linearized equations equations of motion of the electrons one obtaines

$$
\begin{gathered}
\frac{\partial v_{x}}{\partial t}=-\frac{e}{m} E_{x}-\omega_{e} v_{y}-i \frac{k_{\perp} T_{e}}{m} \frac{\widetilde{n_{e}}}{n_{e 0}} \\
\frac{\partial v_{y}}{\partial t}=-\frac{e}{m} E_{y}+\omega_{e} v_{x} \\
0=-\frac{e}{m} E_{z}-i \frac{k_{\|} T_{e}}{m} \frac{\widetilde{n_{e}}}{n_{e 0}}
\end{gathered}
$$

where $\omega_{e}=e B_{0} / m c$ is the electron cyclotron frequency, $T_{e}$ is the electron temperature. In equation (12) the electrons are assumed to be inertialess along the field $B_{0}$, which corresponds to the right hand side of the inequality (9). Then the continuity equation for the electrons turns out to be equivalent to the equation for a perturbation of the electron density derived from the latter equation:

$$
\frac{\widetilde{n_{e}}}{n_{e 0}}=i \frac{e}{k_{\|} T_{e}} E_{z}
$$

The ion equations of motion are written as

$$
\begin{gathered}
\frac{\partial V_{x}}{\partial t}=\frac{e}{M} E_{x}+\omega_{i} V_{y}, \\
\frac{\partial V_{y}}{\partial t}=\frac{e}{M} E_{y}-\omega_{i} V_{x}, \\
\frac{\partial V_{z}}{\partial t}=\frac{e}{M} E_{z},
\end{gathered}
$$


where terms with thermal pressure are neglected in virtue of the left inequality (9). The continuity equation for the ions couples their density perturbations with variations of both longitudinal and transversal velocities:

$$
\frac{\partial}{\partial t}\left(\frac{\widetilde{n_{i}}}{n_{i 0}}\right)+\frac{\partial V_{x}}{\partial x}+\frac{\partial V_{z}}{\partial z}=0
$$

Contrary to the electron and ion components dust relaxes to thermal equailibrium much slower because of low frequency of collisions. Therefore one can assume that in the region where the elctrons and ions are isotropic the dust component is anisotropic. In such conditions in the equations of motion of the dust the anisotropic pressure tensor has to be explicitly used. The linearized gradient of the pressure tensor is [14]

$$
\begin{array}{r}
\nabla P=\widehat{x}\left[\frac{\partial p_{\perp}^{\prime}}{\partial x}+\frac{\partial}{\partial z}\left(p_{\|}-p_{\perp}\right) \frac{B_{x}}{B_{0}}\right]+ \\
+\widehat{y} \frac{\partial}{\partial z}\left(p_{\|}-p_{\perp}\right) \frac{B_{y}}{B_{0}}+\widehat{z}\left[\frac{\partial}{\partial x}\left(p_{\|}-p_{\perp}\right) \frac{B_{x}}{B_{0}}+\frac{\partial p_{\|}^{\prime}}{\partial z}\right] .
\end{array}
$$

Here $p_{\|}=n_{d o} T_{\|}, p_{\perp}=n_{d o} T_{\perp}, n_{d 0}$ is dust density, $T_{\|}$and $T_{\perp}$ its temperature along and perpendicular to the magnetic field, respectively, $p_{\|}^{\prime}, p_{\perp}^{\prime}$ are small deviations of pressure components from the equilibrium values. It has to be mentioned that since the field line is determined by superposition of the external and the wave field, the pressure $p_{\|}$and $p_{\perp}$ in different points should have different projections on to $x$ and $z$ axes. Then the motion equations of dust get the form

$$
\begin{aligned}
\frac{\partial w_{x}}{\partial t}= & -\frac{Z e}{m_{d}} E_{x}-\omega_{d} w_{y}-i \frac{k_{\perp}}{m_{d} n_{d 0}} p_{\perp}^{\prime}- \\
& -\frac{i k_{\|}}{m_{d}}\left(T_{\|}-T_{\perp}\right) \frac{B_{x}}{B_{0}} \\
\frac{\partial w_{y}}{\partial t}=- & \frac{Z e}{m_{d}} E_{y}+\omega_{d} w_{x}-\frac{i k_{\|}}{m_{d}}\left(T_{\|}-T_{\perp}\right) \frac{B_{x}}{B_{0}}, \\
\frac{\partial w_{z}}{\partial t}= & -\frac{Z e}{m_{d}} E_{z}-\frac{i k_{\perp}}{m_{d}}\left(T_{\|}-T_{\perp}\right) \frac{B_{x}}{B_{0}}- \\
& -\frac{i k_{\|}}{m_{d} n_{d 0}} p_{\|}^{\prime},
\end{aligned}
$$

where $\omega_{d}=Z e B_{0} / m_{d} c$ is the dust cyclotron frequency.

In order to determine the perturbations of pressure $p_{\|}^{\prime}$ and $p_{\perp}^{\prime}$ we use the following equations

$$
\begin{aligned}
& \frac{\partial p_{\|}^{\prime}}{\partial t}+p_{\|} \nabla \cdot \mathbf{w}+2 p_{\|} \nabla_{\|} w_{z}=0, \\
& \frac{\partial p_{\perp}^{\prime}}{\partial t}+2 p_{\perp} \nabla \cdot \mathbf{w}-p_{\perp} \nabla_{\|} w_{z}=0,
\end{aligned}
$$

equivalent to the Chew-Goldberger-Law (CGL) invariants, as well as the dust continuity equation 


$$
\frac{\partial}{\partial t}\left(\frac{\widetilde{n_{d}}}{n_{d 0}}\right)+\frac{\partial w_{x}}{\partial x}+\frac{\partial w_{z}}{\partial z}=0
$$

It has to be mentioned that the pressure components $p_{\|}, p_{\perp}$ in equations (22) and (23) are connected with both variation of density and perturbation of temperature - the latter having adiabatic character in CGL theory. As a result, the equation for curl $\mathbf{B}$ in linear approximation is written as

$$
(\nabla \times \mathbf{B})_{x, y}=\frac{4 \pi e}{c}\left(n_{i 0} V_{x, y}-n_{e 0} v_{x, y}-Z n_{d 0} w_{x, y}\right)
$$

\section{THE DISPERTION EQUATION}

\section{A. Derivation of the dispersion equation}

The equations (10)-(12), (14)-(17), (19)-(25) and (7) represent a full set of equations of our problem. In general, this system of equations described the two types of lowfrequency electromagnetic waves: Alfvén and magnetosound. Our goal is to demonstrate that behind the shock wave mirror instability develops in the dust component. It is well known that mirror instability is connected with magnetosound electromagnetic field with the wavevector complanar to the external magnetic and the wave magnetic fields. According to our choice $k_{\perp}$ points along $x$-axis. Hence only the field components $E_{y}, B_{x}$ (and $B_{z}$ ) are connected with the magnetosound wave, while the components $E_{x}$ are $B_{y}$ small. In such conditions the solution of the motion equations (10)-(11), (14)-(15) and (19)-(20) for the Fourier components $\sim \exp i\left(k_{\perp} x+k_{\|} z-\omega t\right)$ in the drift approximation $\left(\omega \ll \omega_{i}, \omega_{d}\right)$ can be written as

$$
\begin{gathered}
v_{x}=c \frac{E_{y}}{B_{0}}, v_{y}=-\frac{c}{B_{0}}\left(E_{x}-\frac{k_{\perp}}{k_{\|}} E_{z}\right), \\
V_{x}=c \frac{E_{y}}{B_{0}}, V_{y}=-\frac{c}{B_{0}}\left(E_{x}+i \frac{\omega}{\omega_{i}} E_{y}\right), \\
w_{x}=c \frac{E_{y}}{B_{0}}, \\
w_{y}=-\frac{c}{B_{0}}\left(E_{x}-i \frac{\omega}{\omega_{d}} E_{y}\right)-\frac{i k_{\|} c}{Z e B_{0}^{2}}\left(T_{\|}-T_{\perp}\right) B_{x}-\frac{i k_{\perp} c}{Z e B_{0} n_{d 0}} p_{\perp}^{\prime}, \\
V_{z}=-\frac{i e}{M \omega} E_{z}, w_{z}=\frac{k_{\perp}}{m_{d} \omega}\left(T_{\|}-T_{\perp}\right) \frac{B_{x}}{B_{0}}+\frac{k_{\|}}{m_{d} \omega n_{d 0}} p_{\|}^{\prime},
\end{gathered}
$$

here the expression for $v_{y}$ is obtained with accounting (13), while in the second equation of (28) the term contained the field component $E_{z}$ is omitted as its contribution into the dispersion equation is small over $Z M / m_{d}$.

The equation for curl $\mathbf{E}$ gives the connection between the field components of interest

$$
E_{y}=\frac{\omega}{k_{\perp} c} B_{z}, B_{x}=-\frac{k_{\|}}{k_{\perp}} B_{z}
$$


From equations (17) and (24) we have

$$
\frac{\tilde{n}_{i}}{n_{i 0}}=\frac{k_{\perp} V_{x}+k_{\|} V_{z}}{\omega}
$$

and

$$
\frac{\widetilde{n_{d}}}{n_{d 0}}=\frac{k_{\perp} w_{x}+k_{\|} w_{z}}{\omega}
$$

With using (27), (29) and (30), it is convenient to rewrite equation (31) in the form

$$
\frac{\widetilde{n_{i}}}{n_{i 0}}=\frac{B_{z}}{B_{0}}+\frac{i k_{\|} e}{M \omega^{2}} E_{z}
$$

In order to derive the perturbation of dust density let us write the equations following from (22), (23) for the Fourier components:

$$
p_{\|}^{\prime}=n_{d 0} T_{\|}\left(3 \frac{\widetilde{n_{d}}}{n_{d 0}}-2 \frac{B_{z}}{B_{0}}\right), p_{\perp}^{\prime}=n_{d 0} T_{\perp}\left(\frac{\widetilde{n_{d}}}{n_{d 0}}+\frac{B_{z}}{B_{0}}\right)
$$

here equations (24), (29) and (30) are accounted. Then with accounting (28), (29) and (34) one obtains from $(32)$

$$
\frac{\widetilde{n_{d}}}{n_{d 0}}=\frac{B_{z}}{B_{0}}\left(1-\frac{k_{\|}^{2}}{\omega^{2}} \frac{3 T_{\|}-T_{\perp}}{m_{d}}\right) /\left(1-\frac{k_{\|}^{2}}{\omega^{2}} \frac{3 T_{\|}}{m_{d}}\right) .
$$

It is obvious that $T_{\perp} / m_{d}$ behind the shock front has the order of the square of the shock velocity, while $T_{\|} / m_{d}$ characterizes the square of the thermal velocity of the dust component before the shock and is therefore negligibly small. Therefore the latter equation can be written as

$$
\frac{\widetilde{n_{d}}}{n_{d 0}}=\frac{B_{z}}{B_{0}}\left(1+\frac{k_{\|}^{2} T_{\perp}}{m_{d} \omega^{2}}\right) .
$$

The field $E_{z}$ can be determined with using the quaineutrality equation $\widetilde{n_{i}}=\widetilde{n_{e}}+Z \widetilde{n_{d}}$ from (13), (33) and (36):

$$
E_{z}=-i \frac{n_{e 0}}{n_{i 0}} \frac{M \omega^{2}}{e} \frac{k_{\|} c_{s}^{2}}{\omega^{2}-k_{\|}^{2} c_{s}^{2}} \frac{B_{z}}{B_{0}}
$$

where $c_{s}=\left(n_{i 0} T_{e} / n_{e 0} M\right)^{1 / 2}$ is the dust ion-acoustic speed. With using the latter equation let us write $v_{y}$ as

$$
v_{y}=-c \frac{E_{x}}{B_{0}}-i \frac{n_{e 0}}{n_{i 0}} \frac{\omega^{2}}{\omega^{2}-k_{\|}^{2} c_{s}^{2}} \frac{k_{\perp} c_{s}^{2}}{\omega_{i}} \frac{B_{z}}{B_{0}} .
$$

The velocity $w_{y}$ can be found from (28), (34) and (36) with accounting the above mentioned interrelation between the longitudinal and transverse dust temperatures: 


$$
\begin{aligned}
w_{y}= & -\frac{c}{B_{0}}\left(E_{x}-i \frac{\omega}{\omega_{d}} E_{y}\right)- \\
& -i \frac{k_{\perp} c T_{\perp}}{Z e B_{0}^{2}}\left(3+\frac{k_{\|}^{2} T_{\perp}}{m_{d} \omega^{2}}\right) B_{z} .
\end{aligned}
$$

Substituting (27), (38) and (39) into the $y$-projection of the curl B we arrive at the dispersion equation

$$
\begin{aligned}
k^{2} V_{A}^{2} & =\omega^{2}\left(1-\frac{\rho_{i}}{\rho} \frac{k_{\perp}^{2} c_{s}^{2}}{\omega^{2}-k_{\|}^{2} c_{s}^{2}}\right)- \\
& -\frac{\rho_{d}}{\rho}\left(3+\frac{k_{\|}^{2} V^{2}}{\omega^{2}}\right) k_{\perp}^{2} V^{2} .
\end{aligned}
$$

Here $V_{A}^{2}=B_{0}^{2} / 4 \pi \rho, V^{2}=T_{\perp} / m_{d}, \rho=\rho_{i}+\rho_{d}, \rho_{i}=M n_{i 0}, \quad \rho_{d}=m_{d} n_{d 0}$.

\section{B. The mirror instability}

In the absence of dust $\left(\rho_{d}=0\right)$ equation (40) is biquadratic

$$
\omega^{4}-k^{2}\left(V_{A}^{2}+c_{s}^{2}\right) \omega^{2}+k^{2} k_{\|}^{2} V_{A}^{2} c_{s}^{2}=0,
$$

and describes fast and slow magnetosonic waves. When dust is present equation (40) is in general bicubic. Similar equation accounting kinetic effects is obtained and analyzed in details in [16]. We consider here the most simple and obvious limiting case when the wave propagates almost perpendicular to the magnetic field: $k_{\|} \ll k \approx k_{\perp}$ which corresponds to a long-wavelength modulation of the magnetosonic waves along the external magnetic field. Then with accounting the condition $\omega / k_{\|} \gg c_{s}$ equation (40) simplifies and becomes

$$
\omega^{4}-A \omega^{2}+B=0
$$

where

$$
A=k^{2}\left(V_{A}^{2}+\frac{\rho_{i}}{\rho} c_{s}^{2}+3 \frac{\rho_{d}}{\rho} V^{2}\right), B=k^{2} k_{\|}^{2}\left(V_{A}^{2} c_{s}^{2}+3 \frac{\rho_{d}}{\rho} V^{2} c_{s}^{2}-\frac{\rho_{d}}{\rho} V^{4}\right) .
$$

The solution of $(42)$ is

$$
\omega^{2}=\frac{1}{2}\left(A \pm \sqrt{A^{2}-4 B}\right) .
$$

The sign "minus" corresponds to the slow magnetosonic wave. Unstable aperiodic solutions for such waves exist if $B<0$ which corresponds to the nonequality

$$
\frac{V^{2}}{c_{s}^{2}}>\frac{3}{2}+\sqrt{\frac{9}{4}+\frac{\rho}{\rho_{d}} \frac{V_{A}^{2}}{c_{s}^{2}}} .
$$


Immediately behind the shock where radiative losses are unimportant the parameter $V^{2}$, which characterizes the post-shock transversal dust temperature, is connected with the shock velocity by the relation $V^{2}=9 v_{s}^{2} / 32$, where $v_{s}$ is the shock velocity; here we assumed explicitle that a dust particle crossing the shock moves with respect to plasma with the velocity $3 v_{s} / 4$. The electron temperature immediately behind the front is $T_{e}=3 m_{e} v_{s}^{2} / 16$, i.e. $V^{2} / c_{s}^{2}=3 m_{i} / 2 m_{e}$. Hence as might be thought the condition (44) fulfils always as $m_{i} / m_{e}>\sqrt{\rho / \rho_{d}}$. In reality, however, $V^{2} / c_{s}^{2}=3 / 2$ is valid up to the region where radiative cooling sets on, since betatron acceleration responsible for an efficient dust destruction establishes far behind the front, where the electron-ion plasma is already isothermal. Therefore, the condition (44) apparently does not fulfil.

In fact, it has to be taken into account that in the domain of radiative cooling all parameters included into (44) vary. Namely, due to radiative cooling and an increase of magnetic field, and caused by it betatron acceleration the orbital velocity of dust particles $V$ grows as $V \propto \sqrt{B}$, or for a frozen-in magnetic field as $V \propto \sqrt{\rho}$. This gives for a cooling plasma where $\rho T \simeq$ const $V^{2} / c_{s}^{2} \propto T^{-2}$; similarly in the radiation domain we have $V_{A}^{2} / c_{s}^{2} \propto T^{-2}$. Therefore, in the domain of radiative cooling the inequality (44) can be written in the form

$$
\frac{3}{2} \frac{T_{0}}{T}>\sqrt{\frac{20}{M_{A}^{2}} \frac{\rho}{\rho_{d}}},
$$

where $T_{0}$ is the plasma temperature immediately behind the shock. Here immediately the shock front we explicitly used the relation $V_{A}^{2} / c_{s}^{2} \simeq 20 / M_{A}^{2}$, where $M_{A}=v_{s} / V_{A 0}$ is the Alfvén Mach number before the shock. Substituting here $T_{0} / T>10$ and a typical for the ISM value $\rho / \rho_{d} \simeq 100$, one can find the condition for the mirror instability $M_{A}>3$, which is deliberately valid for the shocks able to provide dust destruction.

\section{CONCLUSION}

In this paper we have considered a possibility for the mirror instability to develop in the dust component behind the shock. Flows behind the fronts of sufficiently strong shock waves $\left(M_{A}>3\right)$ turn out to be unstable against the mirror instability. Consequently, a laminar magnetohydrodynamic flow behind the shock front supporting betatron acceleration of dust particles becomes violated. One can expect that it causes weakening of the betatron acceleration. Indeed, the mirror instability on nonlinear stages is known to result, in particular, in formation of magnetic voids, holes and growing loops swelling out behind the front to a few dust gyration radii [17], which in our conditions is $r_{c d} \sim 0.3 \mathrm{pc}$ for typical parameters of dust particles and the shock velocity $100 \mathrm{~km} \mathrm{~s}^{-1}$. As a result, charged dust particles are expected to be expelled into the domains of weak magnetic field and decelerated as $p_{\perp}^{2} \propto B$.

It follows from (43) that the characteristic time of the instability has an order

$$
\tau_{m} \sim \sqrt{\frac{\rho}{\rho_{d}} \frac{\lambda}{v_{s}}},
$$

where $\lambda$ is the perturbation wavelength. Assuming the wavelength to be equal to the thickness of the adiabatic SN remnant $\lambda \sim \Delta R \sim 0.1 R_{s}$ and $\rho / \rho_{d}$ equal to a typical in the ISM value 100 , one can find $\tau_{m}$ to be of the order of the remnant age. This estimate is an upper limit of the growth time - short-wavelength perturbations grow obviously faster. The perturbation wavelength is restricted from below by the kinetic limit and by the size 
of the nonuniformity of the post-shock flow. The latter corresponds to the characteristic cooling length. The radiative cooling time can be estimated as $\tau_{c} \sim k T / \Lambda n \sim 10^{11} \mathrm{~s}$ for temperature $T \sim 3 \times 10^{5} \mathrm{~K}$, typical for the beginning of the radiative stage, and density $n \sim 1 \mathrm{~cm}^{-3}$; the corresponding scale is $\lambda \sim 0.3 \mathrm{pc}$. The size of the remnant with the expansion velocity $\sim 100 \mathrm{~km} \mathrm{~s}^{-1}$ in a medium with mean density $n \sim 1 \mathrm{~cm}^{-3}$ is $30 \mathrm{pc}$, hence the lower estimate of the characteristic time of the mirror instability on these stages is of $1 \%$ the remnant age. It can be expected therefore that the instability being developed in the whole possible wavelength range will efficiently restrict the rate of dust destruction. As mentioned above that at nonlinear stages the mirror instability results in breaking up of a regular flow with magnetic field parallel to the front, and leads essentially to formation of loop-like domains with a decreased magnetic field - magnetic voids [17]. It allows to estimate the factor of weakening of dust destruction assuming that magnetic field behind the shock does not increase due to radiative cooling, but in average remains rather the same as if the compression due to cooling would be absent. The corresponding decrease of the destruction efficiency would become an order of magnitude in the range of the "danger" velocity interval $100 \mathrm{~km} \mathrm{~s}^{-1}<v_{s}<300 \mathrm{~km} \mathrm{~s}^{-1}$. Firm estimates can be obtained only from numerical modeling of the instability.

This work is supported by the Russian Foundation of Basic Reserach (project codes 08-02-00933 and 09-02-97019), and in part by Deutsche Forschungs Gemeinschaft under the project SFB 591.

[1] B. T. Draine and E. E. Salpeter, Astrophys. J. 231, 438 (1979).

[2] C. F. McKee, in: Interstellar Dust, eds L. J. Allamandola and A. G.G. M. Tielens (Dordrech: Kluwer, 1989), p. 431.

[3] G. Wallerstein and G. R. Knapp, Ann. Rev. Astron. and Astrophys. 36, 369 (1998).

[4] A. G.G. M. Tielens, in: Formation and Evolution of Solids in Space, eds J. M. Greenberg, A. Li (Kluwer, 1999), p. 331.

[5] G. R. Knapp, in: Tetons 4: Galactic Structure, Stars and the Interstellar Medium, ASP Conf. Ser. 231, 127 (2001).

[6] B. D. Savage and K. R. Sembach, Ann. Rev. Astron. and Astrophys. 34, 279 (1996).

[7] J. E. O'Donnel and J. S. Mathis, Astrophys. J. 479, 806 (1997).

[8] L. Blitz, in: The Evolution of the Interstellar Medium, ed. L. Blitz, ASP Conf. Ser. 12, 273 (1990).

[9] M. H. Heyer and S. Terebey, Astrophys. J. 502, 265 (1998).

[10] J. L. Linsky, B. T. Draine, H. W. Moos, et al., Astrophys. J. 647, 1106 (2006).

[11] D. Breitschwerdt and M. A. de Avillez, Astron. and Astrophys. 452, L1 (2006).

[12] C. G. Seab and J. M. Shull, Astrophys. J. 275, 652 (1983).

[13] A. Kopp, A. Schröer, G. T. Birk, and P. K. Shukla, Phys. Plasmas 4, 4414 (1997).

[14] N. A. Krall and A. W. Trivelpiece, Principles of Plasma Physics (McGraw-Hill, 1973).

[15] B. Abraham-Shrauner, J. Plasma Phys. 1, 361 (1967).

[16] V. V. Prudskikh, L. V. Kostyukova, and Yu. A. Shchekinov, Phys. Plasmas (2009, in press).

[17] M. G. Kivelson and D. J. Southwood, J. Geophys. Res. 101, 17365 (1996). 